\title{
Labyrinthe
}

$22 \mid 2005$ (3)

La Biopolitique (d')après Michel Foucault

\section{De Hölderlin à Marx : mythe, imitation, tragédie}

Entretien réalisé par Bruno Duarte

Philippe Lacoue-Labarthe

\section{CpenEdition}

Journals

Édition électronique

URL : http://journals.openedition.org/labyrinthe/1484

DOI : $10.4000 /$ labyrinthe. 1484

ISSN : 1950-6031

Éditeur

Hermann

Édition imprimée

Date de publication : 1 novembre 2005

Pagination : 121-133

\section{Référence électronique}

Philippe Lacoue-Labarthe, "De Hölderlin à Marx : mythe, imitation, tragédie », Labyrinthe [En ligne], 22 | 2005 (3), mis en ligne le 22 juillet 2008, consulté le 01 mai 2019. URL : http://

journals.openedition.org/labyrinthe/1484; DOI : 10.4000/labyrinthe.1484 


\section{DE HÖLDERLIN À MARX : MYTHE, IMITATION, TRAGÉDIE}

\section{Entretien avec PhiLIPPe LACOUE-LABARTHE réalisé par BRUNO DUARTE}

L'œuvre de Friedrich Hölderlin occupe depuis longtemps une place d'exception dans la pensée du philosophe Philippe Lacoue-Labarthe. On pourrait aller jusqu'à dire de son travail sur le poète allemand qu'il apparaît comme le point d'intersection de deux problèmes fondamentaux: d'un côté, le théâtre, la question de la représentation et de la mimésis; de l'autre, une position critique inépuisable face à la philosophie de Heidegger et au rapport général reliant l'art, la philosophie et la politique.

En 1978, Philippe Lacoue-Labarthe traduit en français la traduction allemande d'Antigone, de Sophocle, faite par Hölderlin, qu'il a mise en scène deux fois au Théâtre national de Strasbourg (TNS), en collaboration avec Michel Deutsch. Cette collaboration se poursuit en 1982 au TNS avec Les Phéniciennes d'Euripide, et en 1990 au centre GeorgesPompidou avec Thermidor, de Michel Deutsch. On compte également parmi ses activités de mise en scène et de dramaturgie La Représentation - Théâtre et Philosophie, au Festival d'Avignon de 1984. Et peu de temps après, il co-écrit et co-met en scène, avec Michel Deutsch, Sit venia verbo (Centre dramatique des Alpes, Grenoble; puis théâtre de la Colline, Paris). En 1998, Philippe Lacoue-Labarthe revient à Hölderlin pour traduire sa version d'CEdipe le tyran, toujours un travail pour le théâtre, cette fois-ci directement lié à la publication de deux textes: Métaphrasis et Le Théâtre de Hölderlin (Puf, 1998).

À son tour, la lecture heideggerienne de Hölderlin, tout comme l'effet d'appropriation et de remythologisation qui s'y trouve impliqué font l'objet des textes qui composent le volume Heidegger - La Politique du poème (Galilée, 2002). Plus récemment, Philippe Lacoue-Labarthe a signé le film Andenken, je pense à vous (collection Proëme, Hors-Oeil, 
2004), essai autour du poème de Hölderlin. Il a en outre participé, avec Bernard Stiegler, Jean-Luc Nancy et Hans Jürgen Syberberg, au film The Ister, de David Barison et Daniel Ross (Australie, 2004), lui aussi centré sur le rapport de Heidegger à Hölderlin.

L'entretien qui suit ne touche pas à l'immense problème posé par l'interprétation heideggerienne de Hölderlin. Il prend plutôt comme point de départ les deux textes de Philippe Lacoue-Labarthe qui font date et que l'on peut lire dans L'Imitation des modernes (Typographies 2), publié en 1986 chez Galilée: «Hölderlin et les Grecs » et « La césure du spéculatif ». Bien que partiellement, il fait référence aussi à quelques-unes des thèses proposées dans les livres La Fiction du politique. Heidegger, l'art et la politique (Christian Bourgois, 1987) et Le Mythe nazi (Éditions de l'Aube, 1991, en collaboration avec Jean-Luc Nancy).

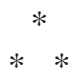

LABYRINTHE - Vous avez traduit les traductions, faites par Hölderlin, d'Antigone et d'Edipe le tyran de Sophocle. Quand et pourquoi exactement?

PhilipPe Lacoue-Labarthe - La première fois, c'était en effet Antigone, vers 1977-1978. La pièce a été représentée deux fois au Théâtre national de Strasbourg, en 1978 et 1979, dans deux mises en scène différentes. Puis, vingt ans plus tard, le TNS m'a demandé la traduction d'Edipe, que j'ai faite en 1997-1998.

LABYRINTHE - Traduire une traduction relève soit de l' imitation, soit d'une violence envers le texte original. Comment avez-vous vécu ce travail, et que signifie, pratiquement, de traduire une traduction?

PhilipPe LACOUe-LABARTHE - Je me suis toujours réglé sur ce que j'avais lu dans l'essai de Walter Benjamin, La Tâche du traducteur: traduire est une grande tâche, qui se confond presque avec la littérature elle-même, mais traduire ce qui est déjà de l'ordre de la traduction n'a aucun sens. Sauf à considérer que les traductions de Hölderlin sont tellement des traductions qu'à la limite elles sont des œuvres de Hölderlin. C'est ainsi que j'ai considéré les choses. Évidemment, j'ai regardé le texte grec, parce que je voulais mesurer l'écart que Hölderlin, volontairement ou pas, avait pris avec lui, mais je traduisais l'allemand, pas le 
grec. Je ne traduisais pas Sophocle, mais plutôt un texte que j'attribuais à Hölderlin, comme si c'était un poème de Hölderlin.

LABYRINTHE - Envisager l'écriture moderne comme traduction ou réécriture revient chez Hölderlin à travailler sur une répétition de ce qui n'a jamais eu lieu dans l'art grec, et qui pourtant y était, comme vous l'affirmez. L'écriture est alors de l'ordre du paradoxe, quelque chose comme une répétition inaugurale, une fondation mimétique originale.

PhilipPe Lacoue-Labarthe - Je crois que l'expérience de ce paradoxe est l'expérience même de la littérature. Toute littérature s'écrit à partir d'une autre littérature. On le voit par exemple dès la tragédie grecque: les grands tragiques écrivent à partir d'intrigues ou de fables - mythoi, comme dit Aristote - léguées par la tradition, écrite ou orale, des poèmes épiques, et ils transforment cela comme ils veulent. Quand on voit les écarts que prennent Sophocle et Euripide à l'égard des mêmes épisodes de la légende et du mythe, c'est impressionnant.

Prenons Les Phéniciennes d'Euripide, par exemple. Edipe, quand ses fils se battent pour le pouvoir à Thèbes et qu'Antigone intervient, est enfermé dans le palais et pas du tout parti en exil. Jocaste est encore vivante, et c'est elle qui essaie de séparer les deux frères et de représenter la justice. Cela veut dire qu'il existait probablement un cycle légendaire ou mythique autour de Thèbes, avec toute la filiation qui engendre finalement Edipe. Il y avait donc une histoire à peu près fixée, des récits, probablement écrits, parce que, contrairement à ce que l'on croit, les grandes épopées ont été écrites relativement tard, y compris celle d'Homère, et c'est à partir de ces scénarios-là que les tragiques travaillaient, en changeant ce qu'ils voulaient changer. C'est la règle de toute littérature: on écrit toujours à partir de quelque chose qui a déjà été écrit.

LABYRINTHE - Après l'échec de La Mort d'Empédocle (1798), Hölderlin érige Edipe en modèle de la tragédie moderne, en édifiant celle-ci comme traduction de la tragédie ancienne. Comment faut-il penser cette transformation?

PhilipPe Lacoue-Labarthe - En effet, Hölderlin ne parvient pas à écrire Empédocle: ne trouvant pas de scénario, à l'époque même où il lisait et relisait la Poétique d'Aristote, il doit se rendre compte que, pour Aristote, une tragédie, c'est une bonne intrigue. Il comprend que son scénario n'est pas bon, parce qu'il est tout simplement l'application d'une 
sorte de fable spéculative au théâtre, ce qui ne peut pas faire du théâtre. Il n'y a pas de conflit, il n'y a aucune intrigue réelle.

Ensuite, je dirais que l'idée de traduire Sophocle, donc de traduire, au sens fort, du grec ancien pour le présenter comme un exemple moderne, c'est une idée de Winckelmann radicalisée. Quand Winckelmann disait: il nous faut imiter les Anciens pour devenir à notre tour inimitables - une proposition des Pensées sur l' imitation des æeuvres grecques en peinture et en sculpture (1755) -, je crois que Hölderlin est le premier à prendre cette sentence au pied de la lettre. Il se rend compte que, dans ce qu'il connaît en tout cas - le classicisme français, le théâtre de Schiller et de Goethe -, à chaque fois il y a une espèce d'adaptation, de modernisation de la tragédie ancienne, sans qu'il y ait encore un effort pour retrouver ce que cette langue, cette dramaturgie pouvaient avoir d'archaïque, d'antérieur à tout ce qui a été ensuite imité par la tradition (toutes les traditions romaines, et ensuite tout ce qui s'est fait après la Renaissance).

Hölderlin veut faire une traduction beaucoup plus fidèle à l'original; avec cette idée, en effet paradoxale, que plus on est fidèle à l'original, plus on colle à la langue de ce genre d'œuvres: plus c'est surprenant historiquement, et plus cela peut produire un choc dans le théâtre moderne, ne serait-ce que parce que Hölderlin essaie de traduire littéralement, même en courant le risque de se tromper. Il essaie de scander et de respecter une prosodie qui est celle des Grecs et qu'il tente d'adapter à la prosodie allemande - ce qui n'est pas facile, et qui force à créer un langage pour le moins incompréhensible pour cette époque. Il dit justement que cela est aussi incompréhensible pour notre époque que cela pouvait l'être pour les Grecs eux-mêmes. Et je crois que c'était son dernier projet, et que cela devrait représenter pour lui une étape décisive dans ce qu'il cherchait à faire, c'est-à-dire produire une littérature vraiment moderne, vraiment dégagée du modèle ancien. Le paradoxe de sa démarche, c'est qu'une extrême fidélité aux œuvres anciennes revient à prendre un maximum de distance par rapport à elles. Plus je suis fidèle aux Anciens, plus je suis moderne: c'est la structure même du paradoxe. Et je crois que c'est exactement ce qu'il cherchait.

LABYRINTHE - L'ambition de créer une écriture moderne n'est-elle pas aussi un geste politique dissimulé?

PhILIPPE LACOUE-LABARTHE - Hölderlin avait à sa disposition trois corpus tragiques; après avoir été tenté par Euripide (Les Bacchantes), il 
n'a retenu que Sophocle, et dans Sophocle il n'a choisi qu'Antigone et Edipe: Antigone pour le tragique ancien et CEdipe pour le tragique moderne. S'il a fait ce choix, il me semble que c'est, pour l'essentiel, en raison de préoccupations politiques modernes. Edipe, en particulier, était un personnage qui refaisait surface dans l'histoire, sous la forme du dictateur de la raison. C'était la Révolution française, Robespierre, Saint-Just, puis ensuite Bonaparte. Les traductions de Hölderlin sont contemporaines de la montée en puissance de Bonaparte, qu'il admirait énormément: il était persuadé que Bonaparte avait pacifié l'Europe et réglé le problème, non pas des États-nations, mais celui des maisons royales, qu'il était parvenu à établir une République universelle, c'est-à-dire, si l'on excepte l'Amérique, européenne. Plus tard, il aura peut-être été déçu, mais au moment où il écrit $E$ dipe, la figure de Bonaparte, du successeur de Robespierre, est encore indécise, mais au bord de la démesure (de l'hybris), et je crois qu'il veut montrer à quel point la tragédie d'Edipe, de cet homme qui croit tout savoir, qui incarne la raison, et qui voudrait incarner un nouveau droit, une nouvelle justice, une souveraineté, que cette figure-là est moderne. Jusque dans sa « folie ».

LABYRINTHE - Cela croise en un sens votre analyse du mythe (dans "La Fiction du politique » et «Le Mythe nazi ») comme fictionnement, façonnement ou présentation d'un modèle, analyse elle-même liée à la question du mimétisme (imitation "originale» des Anciens) comme production de l'identité de tout un peuple, à savoir l'Allemagne. Il reste pourtant à définir la portée de ce mécanisme. S'agit-il d'une dimension politique circonscrite ou d'un principe universel soumis aux lois de la tragédie?

PhilipPe LaCoue-Labarthe - Je dirais que c'est plutôt universel. Mes analyses sur l'Allemagne concernent un contexte où il se trouve qu'on a fait grand cas, pendant plus d'un siècle, de la tragédie, et où il y a un enjeu politique visible dans les œuvres qu'on a pu produire, quel que soit l'axe politique choisi. Quand les Allemands, même à partir de Goethe, dès la fin du XVIII' siècle, s'emparent du matériau grec, c'est pour parler de l'Allemagne, pas de la situation européenne. Mais, en même temps, c'est universel: le mythe a toujours fonctionné comme un dispositif chargé de procurer une identité à ceux qui y adhèrent, d'une manière ou d'une autre. Et adhérer au mythe, cela veut dire se laisser dicter par lui, consciemment ou inconsciemment, les conduites pratiques de l'exis- 
tence. Cela ne signifie nullement que le mythe n'est qu'un réservoir de recettes. Il donne aussi des exemples, comme ceux du courage, de la douleur, de la lâcheté, etc. Le mythe donne des sortes de cadres praxiques à l'existence. Il est en réalité, pour une grande part, produit à cet effet.

LABYRINTHE - Prenons le concept d'imitation ou de mimétologie historique. Le caractère indissociable du mythe et de l' histoire tient-il à un mouvement par lequel l'histoire s'introduit dans le mythe et le déforme?

PhilipPe Lacoue-Labarthe - Je crois plutôt l'inverse. C'est le concept de mythe qui gouverne le concept d'histoire. Marx remarquait, dans un passage célèbre de son pamphlet sur le Dix-Huit Brumaire de Louis Bonaparte (1851), que les événements historiques qui, pour la première fois, se jouent comme une tragédie, dès qu'ils se répètent, apparaissent comiques. La répétition dans l'histoire, aussi atroce puisset-elle être, est une comédie. Cette vérité ne s'est pas démentie.

Marx citait, pour exemple, la pose à l'antique, spartiate ou romaine, des révolutionnaires de 1789. Mais, aussi bien, 1917 répète 1792 . Lénine prend le pouvoir sur le modèle de la dictature montagnarde (ce qu'on appelait la Montagne, à la Convention), c'est-à-dire sur le modèle robespierriste. Hitler est nourri de néo-hellénisme à l'allemande, et dès qu'il prend le pouvoir, il accomplit son identification à Périclès. Tel est son vrai modèle. De même, Mussolini se prend pour César. Et l'on voit à quel point - dans les arts, dans les costumes, dans l'architecture, dans l'organisation des fêtes, dans les jeux, dans les formes de militarisation de la société - on a des modèles historiques devenus mythiques qui sont agissants. Hitler, c'est un mélange du caractère somptueux de l'Athènes de Périclès et de la rigidité militariste de Sparte. Ce mélange-là date de Rousseau. Et après la guerre, certaines choses sont véritablement attristantes. Déjà Napoléon s'était fait couronner empereur sur le modèle de ceux du Saint Empire romain germanique.

$\mathrm{Au}$ moment de la décolonisation, des dictateurs africains prennent le pouvoir, toujours sur le modèle jacobin, léniniste, suivant la révolution militaire. L'un d'eux s'est même couronné empereur, imitant Napoléon. Tout cela peut continuer longtemps. À la fin, ne restent plus que des imitations d'imitations. Le processus ne cesse de se dégrader. Combien de dictateurs sud-américains ont imité Bonaparte, à commencer par Bolivar? 
LABYRINTHE - Mais le schéma de l'imitation historique rendrait alors possible toute analogie concernant l' histoire du XXe siècle. Celui par exemple qui rapproche l'imitation des Anciens de l'imitation de Marx: il s'agirait d'imiter le marxisme comme s'il n'avait pas eu lieu, sachant pourtant que quelque chose y était posé par Marx. Il serait donc question de produire l' imitation originale, le mimétisme premier comme inauguration du marxisme.

Philippe Lacoue-Labarthe - Cela relève d'une attitude politique générale qui définit à mes yeux ce qu'on a appelé très vaguement l'ultragauchisme européen - parce que l'on a mis beaucoup de choses hétérogènes sous ce nom. L'ultra-gauchisme européen a toujours consisté à dire ceci: il y a dans Marx une vérité que Marx n'a pas réussi à énoncer, elle y est, et c'est à nous de la trouver. Alors que, jusqu'à présent, on n'a fait qu'exploiter des vérités partielles qui étaient explicites dans Marx et qui ont eu des conséquences catastrophiques: Lénine, Trotski, Staline...

Cette ligne politique se résume ainsi: il n'y a jamais eu de communisme, il faut l'inventer, il est caché dans Marx, il est à venir. L'attitude de certains marxistes allemands, d'une certaine gauche radicale allemande, me fait beaucoup penser à ce qu'était le côté révolutionnaire de la Réforme, non pas la Réforme instituée par Luther, mais plutôt la gauche de la Réforme qui se confondait avec le mouvement piétiste, c'est-à-dire ce mouvement dans lequel ont été élevés Schiller, Hölderlin, Hegel, Schelling. Ils sont tous des enfants du piétisme politique. Le piétisme revenait à radicaliser Luther: de même que Luther disait que le catholicisme avait déformé le message chrétien, eux accusaient Luther d'avoir encore déformé le message chrétien. Ce message était donc encore quelque chose de caché, d'ésotérique, mais qu'il fallait retrouver. En ce sens-là, si l'on fait de Marx une sorte de Luther moderne, ce qui n'est pas faux, à bien des égards, c'est en effet un mouvement mystique.

LABYRINTHE - Que voulez-vous dire quand vous affirmez que l' effondrement de la chrétienté est un effondrement du théologico-politique?

PhilipPe LaCoue-LabarTHE - J'appelle chrétienté l'organisation du pouvoir et de la souveraineté, en Occident, à partir de la conversion de l'empereur romain au christianisme. Une sorte de soutien théologique est apporté au politique. Les souverains sont dits souverains de droit divin par la grâce de Dieu. 
La chrétienté commence, admettons-le, quand l'empereur Constantin se convertit au christianisme. Il y a eu évidemment beaucoup de résistances, en particulier en Allemagne, qui correspondent à la révolte luthérienne quelques siècles plus tard, puisque c'est toujours le nord de l'Allemagne qui se réveille contre Rome, l'Église et les pouvoirs politiques, et engage le processus de la Réforme. Malgré ces résistances, il y a bien eu une Europe chrétienne, dont l'unité relative était fondée sur la référence au christianisme. Mais le Capital a commencé à la détruire $\mathrm{au} \mathrm{XV}^{\mathrm{e}}$ siècle. Des princes, des propriétaires terriens et la bourgeoisie commerçante se sont révoltés contre la confiscation ecclésiale de la richesse et de la circulation monétaire, contre certains interdits économiques - touchant le prêt, l'intérêt, l'endettement - que justifiait une idéologie officielle de la pauvreté et de la charité. Cette rébellion économique, marchande au fond, s'est accompagnée et soutenue d'une manière de « révolution culturelle » (la Renaissance) et a commencé à saper les bases du christianisme politique: papauté, restes du Saint Empire romaingermanique, royautés, etc. Ce fut le premier grand coup porté contre la domination chrétienne, catholique, sur le politique. Il est venu, entre autres, des Médicis, des banquiers italiens, génois et espagnols; puis a eu lieu la conquête de l'Amérique du Sud, et ensuite la Réforme, qui casse la relative homogénéité du christianisme, en Europe de l'Ouest et même en Europe centrale. À ce moment-là, l'hégémonie chrétienne est mise en cause.

LABYRINTHE - Ce serait donc la substitution directe d'une théologie politique par une économie politique.

PhillPPe Lacoue-LABARTHE - Oui, tout à fait. Moyennant toutefois la « révolution culturelle » que j'évoquais à l'instant: Renaissance, retour à l'antique, émancipation de la pensée, libération spirituelle (eu égard au dogmatique ou au canonique), inventivité scientifique, mutation de l'art, etc.

LABYRINTHE - Mais cet effondrement est-il nécessairement un accomplissement, une situation définitive?

PhilipPe Lacoue-Labarthe - Je crois que la chrétienté est finie. Sauf à reparaître alors, d'une manière étrange, à partir d'une version du protestantisme, qui est la version anglo-américaine, car les États-Unis sont le dernier État théologique puissant où la théologie ne soit pas simplement 
une révolte, comme dans les pays musulmans. C'est encore une théocratie, me semble-t-il.

LABYRINTHE - Pour reprendre tout le raisonnement, tel qu'on le trouve dans un de vos textes : à l' effondrement de la chrétienté ou du théologico-politique succède la lutte entre la religion politique et la religion de l'art. Le résultat en serait la modernité, comprise comme exigence de fondation et d'Absolu, à la fin du XVIII siècle et pendant une partie du $X I X^{e}$. Pour isoler un quatrième mouvement dans ce cycle, la synthèse des deux religions pourrait s'accomplir, au XXe siècle, comme une espèce de tragédie formalisée ou retraduite par le concept de barbarie ou de désastre. Est-ce exact?

PhilipPe LACOUE-LABARTHE - Je ne dirais pas barbarie, mais plutôt « désastre », parce que « barbarie » renvoie encore au concept grec. $\mathrm{Ou}$ alors il faut prendre le concept de barbarie au sens de Lévi-Strauss : est barbare une civilisation qui trahit ses propres idéaux. En ce sens précis, on pourrait dire que le nazisme est une barbarie.

Le désastre retraduit peut-être le concept de tragique, mais pas celui de tragédie. La tragédie, c'est la représentation du tragique; le tragique, c'est la situation qui est représentée par la tragédie. Mais en même temps cela n'est pas séparable pour l'analyse: on ne voit le tragique à l'œuvre que dans les tragédies. Quand Peter Szondi dit que depuis Aristote il y avait une poétique de la tragédie et depuis Schelling une philosophie du tragique, je ne peux pas être d'accord. Je crois que la philosophie du tragique qui existe en effet est encore forcément une poétique de la tragédie, encore un commentaire d'Aristote. On ne peut pas séparer les deux.

LABYRINTHE - Revenons encore sur ce point. L'absence de fondement et la volonté d' identification qui succèdent à l' effondrement d' une transcendance chrétienne, vous les décrivez comme l'histoire d'un fictionnement qui consiste à imiter l'imitation (romaine ou française) des Anciens, en remarquant qu'une telle imitation au second degré relève d'une volonté d'art. Mais on pourrait dire de ce dessein esthétique/politique qu'il est encore de l'ordre de la transcendance.

PhilipPe LACOUE-LABARTHE - Chaque fois qu'il y a des fondations de grands empires, de grandes entités politiques, se produit toujours un énorme investissement dans l'art. Périclès a fait Athènes, c'est sous 
Périclès que l'art athénien a été florissant. Il a fait d'Athènes une œuvre d'art, puisque c'était cela l'image même du pouvoir et de la souveraineté.

Il n'y a qu'une puissance qui agit dans l'Histoire sans recours à la transcendance, c'est le Capital. Mais il subsiste, en même temps, un dysfonctionnement américain, puisque le pouvoir américain se réclame d'une sorte de théologie, aussi confuse soit-elle. Cette espèce d'autofreinage de l'extension du capital américain est assez étrange, si l'on considère que le Capital constitue la seule immanence. La seule machine historique qui soit totalement immanente, c'est la machine financière.

LABYRINTHE - Toujours suivant votre description: imiter les Anciens suppose une appropriation du propre de l'être grec, l' inimitable même, du fait qu'il n'a jamais eu lieu. Il faut faire l' expérience de la différence et du dépaysement pour atteindre l' appropriation de soi. Le risque serait alors de sombrer dans la schizophrénie. Quels sont les termes de cette appropriation?

PhilipPe Lacoue-Labarthe - L'art est toujours l'invention d'un impropre, pour arriver à une appropriation. Il s'agit d'introduire le propre dans l'impropre, et de montrer ce conflit même. Cela fonctionne comme le paradoxe: plus je me déproprie pour m'approprier, moins j'arrive à m'approprier, ou plus je me déproprie de fait. Ce mécanisme, qui serait idéalement un mécanisme dialectique effectif, il est impossible qu'il « réussisse ». Et la grande aventure de l'art moderne est d'avoir compris cet impossible, d'avoir subi cette impossibilité. S'il y a tant de fous, de « cas » pathologiques - Hölderlin, Poe, Lenz, puis Nietzsche, Artaud dans cette époque, cela veut dire que la machine « je me déproprie pour m'approprier » est enrayée. C'est le mécanisme même de la folie.

Hölderlin et Nietzsche nous ont dit que nous n'avons plus rien à faire avec les Grecs. Ils sont allés jusqu'au bord d'une espèce de gouffre, et c'est ce qu'ils nous ont légué. Je ne dis pas que ce qu'il faut imiter est la psychose, mais la structure psychotique reste malgré tout ce qui nous domine. La névrose, c'est le Capital, qui gère cela très bien. La psychose, qu'elle soit individuelle ou étatique, c'est autre chose: elle n'est pas gérée.

LABYRINTHE - Vous parlez de l'expérience tragique moderne comme d' une "errance de l' impensable». L'impropre est-il cet impensable? 
PhilipPe Lacoue-Labarthe - On peut le dire. La formule, qui vient de Hölderlin, désigne le destin d'EEdipe. L'impensable tient peut-être dans cette question: pourquoi ne suis-je pas proprement moi-même? Ce qui est le cas d'Edipe par excellence.

LABYRINTHE - Chez Pasolini, par exemple, on trouve ce concept de rage qui détermine tous les éléments de la tragédie pour une expérience moderne : la sainteté irréligieuse, l' accouplement de la brutalité et de la beauté, etc. Mais à quelle réalité appartient ce concept, s'il n'est ni religieux ni politique?

PhilipPe LACOUE-LABARTHE - C'est encore un concept religieux: la colère. Chez Pasolini, un des rares artistes presque authentiquement chrétiens de ce siècle, c'est évident. Sa force lui vient d'une dernière vie primitive chrétienne, une colère contre ce monde, contre l'injustice, au nom d'un amour de l'humanité. De même, ce qui anime Marx, enfant de la Réforme marqué par le piétisme radical que j'évoquais tout à l'heure, c'est la colère. Une colère de l'Ancien Testament, comme celle des prophètes, quand ils voient l'état où se trouve Israël. Cette colère définit une sainteté, qui est l'attitude prophétique. À l'origine de cette idée, on trouve les textes du grand prédicateur Oettinger, qui a eu tant d'influence sur Schiller, Schelling, Hölderlin. Oettinger est une figure majeure du piétisme, qui est un mouvement politique, puisque son mot d'ordre est révolutionnaire. Depuis Jakob Böhme, on exigeait une Réforme généralisée. Mais pour les piétistes, la Réforme a été seulement partielle, et fut par conséquent un échec. De même, plus tard, le communisme apparâ̂tra comme un échec, car l'établir dans un seul pays, cela ne veut rien dire. Il fallait donc généraliser la Réforme. Or, ce mot d'ordre a été celui des jeunes républicains souabes élevés dans la théologie, dont Marx dérive en droite ligne. Il y a là une Stimmung proprement vétéro-testamentaire, comme disent les théologiens, celle de la colère. Ou bien le Dieu d'Israël est un Dieu qui se met en colère et celle-ci devient sa manifestation même; ou bien les prophètes arrivent comme des personnages étranges, non pas pour dire l'avenir, mais pour s'adresser au peuple, et, dans la colère, l'accuser. Comme Moïse, comme Jérémie se mettent en colère. La Stimmung de la sainteté, c'est la colère et donc l'amour, c'est la colère.

LABYRINTHE - Ce concept de piétisme politique, médiatisé par celui de la colère, permet donc de resituer Marx dans la lignée de Hölderlin. 


\section{Labyrinthe, $n^{\circ} 22$}

PhilipPe LACOUE-LABARTHE - Oui. Le seul numéro de cette revue que Marx a fondée lors de son arrivée à Paris, les Annales franco-allemandes, contient une épigraphe tirée d'Hypérion, de Hölderlin. Il s'agit de la fameuse lettre finale d'Hypérion à Bellarmin (l'avant-dernière du roman). Hypérion est aussi un roman écrit dans la colère. Il y a là une affiliation directe.

LABYRINTHE - Et Heine, par exemple, se situerait-il aussi dans une telle lignée?

PhilipPe LaCoue-Labarthe - Oui. L'Histoire de la religion et de la philosophie en Allemagne (1852) de Heinrich Heine a beaucoup compté pour Marx.

C'est un livre prodigieux, où l'on saisit qu'il n'y a de philosophie allemande qu'à partir du passage de Luther dans la théologie allemande en général. Marx l'a bien compris. En particulier, c'est la langue de Luther, la langue des sermons et des traités, comme de la traduction biblique, qui a formé la langue philosophique allemande. La démonstration de Heine est éblouissante.

LABYRINTHE - Cette lignée d'enragés produit-elle encore des héros tragiques, au sens de Hegel, des sujets dont l'expérience est une innocence coupable?

PhILIPPE LACOUE-LABARTHE - Oui. Enragé est le bon mot, parce que les seuls révolutionnaires que Hölderlin ait rencontré à Paris - s'il est passé par Paris, ce que je crois - sont des enragés. Le concept de l'innocence coupable est ce qui définit (Edipe. Il est coupable de ce qu'il a fait, mais il ne le savait pas, donc il est innocent. Son destin est un oxymore: innocent-coupable, contradiction sans solution.

LABYRINTHE - Heiner Müller a parlé une fois du tragique moderne comme d'une expérience où l'on peut vivre sans espoir et sans désespoir, ce qui revient à se nourrir de l'effet tragique - la «catharsis»comme d'un flux d'énergie: pour que l'un reprenne ses forces, l'autre doit s'épuiser.

PhilipPe LaCoue-Labarthe - C'est encore une conception aristotélicienne de la tragédie, tout de même. Elle fonctionne à condition que l'effondrement de l'autre soit représenté. Ce qui est évidemment le cas chez Müller. 
LABYRINTHE - L'image de la césure ou d'une interruption de la logique dialectique spéculative, dont il est question dans un des essais de «L'Imitation des Modernes» sur Hölderlin, est-elle à comprendre comme un mécanisme fondateur qui ne concerne que la seule tragédie, ou faut-il plutôt y voir un énoncé historique à part entière?

PhILIPPE LACOUE-LABARTHE - Le hiatus découvert par Hölderlin au sein même du processus dialectique ne pouvait que jouer sur l'idéalisme spéculatif, et à terme le ruiner, ce que Heidegger a très bien vu. Je répondrai un peu comme Heiner Müller là-dessus : l'énoncé qui fait césure ne peut être qu'un énoncé qui vient des morts. Qui sont les morts, aujourd'hui? Que considère-t-on comme les morts qui pourraient énoncer quelque chose faisant coupure? Je crois que, dans l'histoire européenne la plus récente, les morts en question sont les Juifs. Mais dans la mesure où cette histoire s'efface, on ne peut plus savoir. Celui qui pourrait porter et prononcer un tel énoncé serait un artiste et personne d'autre, à la limite un philosophe, mais en tout cas il faudrait que ce soit le porte-parole des morts - ce que Müller a essayé de faire. Je nomme les Juifs à cause de l'enjeu théologico-politique qui s'est créé en Europe. Mais on peut élargir le concept à toutes les victimes de la fin du politique moderne.

LABYRINTHE - Il faudrait alors revenir au parallélisme entre la tragédie et l'injustice, entendu comme concept universel. Il reprend ou resitue la continuité entre la tragédie et la politique.

PhilipPe Lacoue-Labarthe - Le concept le plus important de la tragédie est celui de $d y k e ́$, la justice. Le lieu de la tragédie a toujours été la politique. Mais la tragédie, en particulier pour Hölderlin, montre précisément que la justice est l'au-delà inaccessible de la politique, et à ce titre (transcendantal, sinon transcendant), la condition - de possibilité ou, peut-être, d'impossibilité - de la politique.

LABYRINTHE - Et la religion?

PhilipPe LaCOUe-Labarthe - Pour moi, c'est de la politique mythologisée. 